\title{
Rauchstopp senkt Mortalität deutlich
}

\author{
Frauen, die um 1940 geboren wurden, gehörten zur ersten \\ Generation, in der viele eine beträchtliche Menge an Zigaretten \\ im Laufe ihres Lebens konsumierten. Daher können die vollen \\ Auswirkungen des anhaltenden Rauchens auf die Mortalität der \\ Frauen erst jetzt im 21. Jahrhundert beobachtet werden. Mit \\ dieser Thematik haben sich K. Pirie et al. beschäftigt. \\ Lancet 2013; 381: 133-141
}

Die Autoren nahmen von 1996-2001 1,3 Mio. Frauen aus Großbrtitannien in ihre prospektive Studie auf und untersuchten sie 3 und 8 Jahre später erneut. 2011 wurde anhand der nationalen Sterberegister überprüft, welche der Teilnehmerinnen zwischenzeitlich verstorben waren. Die Frauen wurden in aktive Raucherinnen und Ex-Raucherinnen unterteilt. Letzere wurden wiederum untergliedert nach dem Alter, in dem sie das Rauchen aufgegeben hatten. Die aktiven und Ex-Raucherinnen wurden mit Frauen, die nie geraucht hatten, korreliert.

Rauchen verkürzt bei Frauen das Leben um mindestens 10 Jahre. Wird das Rauchen im jungen Alter aufgegeben, sinkt die Mortalität erheblich, so die Autoren. 100000 Frauen wiesen zu Studienbeginn bereits eine Vorerkrankung auf und wurden aus der Analyse ausgeschlossen. Die übrigen 1,2 Mio. waren zum Aufnahmezeitpunkt im Mittel 55 Jahre alt. Von ihnen waren $20 \%$ aktive Raucherinnen, $28 \%$ Ex-
Raucherinnen und 52\% hatten nie geraucht. $6 \%$ der Studienpopulation starben im mittleren Alter von 65 Jahren. Nach 12 Jahren lag die Mortalitätsrate bei Raucherinnen 2,76-mal höher (95\%-Konfidenzintervall [KI] 2,71-2,81) als bei Nichtraucherinnnen, obwohl 44\% der Raucherinnen zu Studienbeginn, die auf die Umfrage nach 8 Jahren antworteten, bis dahin mit dem Rauchen aufgehört hatten. Selbst wenn die Frauen zu Studienbeginn weniger als 10 Zigaretten pro Tag rauchten, war die Mortalität verdoppelt (Rate Ratio 1,98, 95\%-KI 1,91-2,04). 23 der 30 häufigsten Todesursachen waren bei Rauchern deutlich erhöht; bei Lungenkrebs betrug das Rate Ratio 21,4 (95\%-KI 19,7-23,2). Frauen, die im Alter von 25-34 bzw. 35-40 Jahren das Rauchen aufgegeben hatten, hatten im Vergleich zu Nichtrauchern eine erhöhte Gesamt- und Lungenkrebs-Mortalität. Verglichen mit kontinuierlichen Rauchern war die Mortalität jedoch um 97\% bzw. 90\% reduziert. Im Mittel verloren aktive Raucherinnen 11 Lebensjahre.

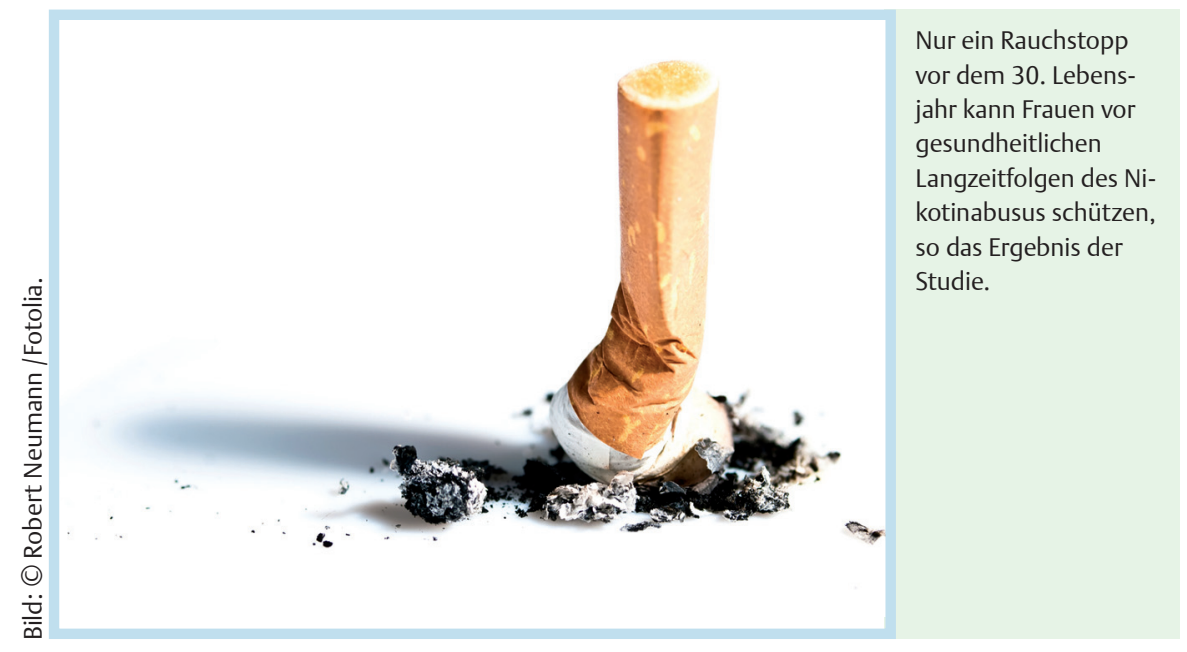

\section{Fazit}

Rauchen verkürzt bei Frauen das Leben um mindestens 10 Jahre. Wird das Rauchen im Alter von unter 40 bzw. unter 30 Jahren aufgegeben, sinkt die Mortalität erheblich, so die Autoren.

\section{Kommentar zur Studie}

R. R. Huxley und M. Woodward kommentieren, dass diese wegweisende Studie nun harte Zahlen liefere, mit denen der lebensverkürzende Einfluss des Rauchens bewiesen werde. Rauchende Frauen verlieren 10 Jahre ihres Lebens. Beenden sie das Rauchen vor dem 30. Lebensjahr, verkürze sich ihr Leben allerdings kaum. Interessant sei v. a. die Beobachtung, dass Rauchen bei Frauen das Mortalitätsrisiko durch eine koronare Herzkrankheit deutlich stärker steigere als dies bei rauchenden Männern der Fall ist, so die Autoren.

Lancet 2013; 381: 96-98

\section{Dr. Christoph Feldmann, Köln}

Nachwuchsförderung

\section{Online-Plattform für junge Pneumologen}

Die Deutsche Gesellschaft für Pneumologie und Beatmungsmedizin e.V. (DGP) arbeitet aktiv an der Nachwuchsförderung und stellt ein neues Webportal vor. Mit www.ich-werde-pneumologe.de möchte die DGP Studierenden und jungen Medizinern das Fachgebiet vorstellen und ihr Interesse wecken. Neben Informationen zur Bedeutung der Pneumologie als Schwerpunktfach der Inneren Medizin werden die Aspekte Beruf und Familie sowie Karrieremöglichkeiten dargestellt. Das Portal bietet hierzu auch die Möglichkeit, nach familienfreundlichen Kliniken, Weiterbildungsstätten und pneumologischen Lehrstühlen in Deutschland zu suchen. Ein weiterer wichtiger Hauptpunkt ist die Verdeutlichung der Komplexität und Vielfältigkeit des Fachgebiets. An Beispielen werden eine Reihe fachübergreifender Krankheitsbilder erläutert, denn eine Atemwegserkrankung betrifft in vielen Fällen nicht nur die Lunge.

Nach einer Mitteilung der DGP 